\title{
Semantic XML Filtering by Ontology Combination
}

\author{
Kyeung Soo Lee, Dong Ik Oh, and Yong Hae Kong \\ Division of Information Technology Engineering, Soonchunhyang University, \\ Asan-si, Choongnam, 336-745, Korea \\ \{kslee, dohdoh, yhkong\} asch.ac.kr
}

\begin{abstract}
An XML document search method which expands XML query through conceptual structuring and association on intra/inter-ontology is proposed. The method can filter out semantic information from various XML documents and can be effective for a large application.
\end{abstract}

\section{Introduction}

Expanding XML queries based on ontology enables filtering out semantic information from various forms of XML documents: [1],[2]. To do this, complete and elaborate ontology is required: [3]. However, building and accessing such ontology is very hard and inefficient. To be effective and efficient in XML document filtering, combining ontology is attempted in expanding XML query. A query expanding algorithm is first developed through conceptual structuring and concept association for intra-ontology. Then, the algorithm is generalized for inter-ontology structuring and association.

As an application example, ontology 'College-Research-Center' is combined with ontology 'Programming-Skills' and 'College-Courses' shown by dotted lines in Fig. 1. $\mathrm{XQL}$ (XML Query Language) is used for query application and the effectiveness of $\mathrm{XML}$ filtering is tested by a sample XML document.

\section{Query Expansion by Ontology Combination}

Expansion by conceptual structuring: Add sub-concepts to XML query. Query '//Person[skill="C']' is expanded to '//(Person I Professor I Engineer I Student I MasterStudent I PhDStudent)[skill="C"]' in 'College-Research-Center'.

Expansion by concept association: Set an association between concepts A and B if attribute of concept A is concept B and vice versa. In 'College-Research-Center', concepts 'Student' and 'Project' are associated as shown by a solid line in Fig. 1(b). The following is the corresponding rule for query expansion.

FORALL Pers1, Proj1

Proj1 : Project[member ->> Pers1] <-> Pers1 : Student[project ->> Proj1]

This research was supported by University IT Research Center Project

J.M. Cueva Lovelle et al. (Eds.): ICWE 2003, LNCS 2722, pp. 395-398, 2003.

(C) Springer-Verlag Berlin Heidelberg 2003 


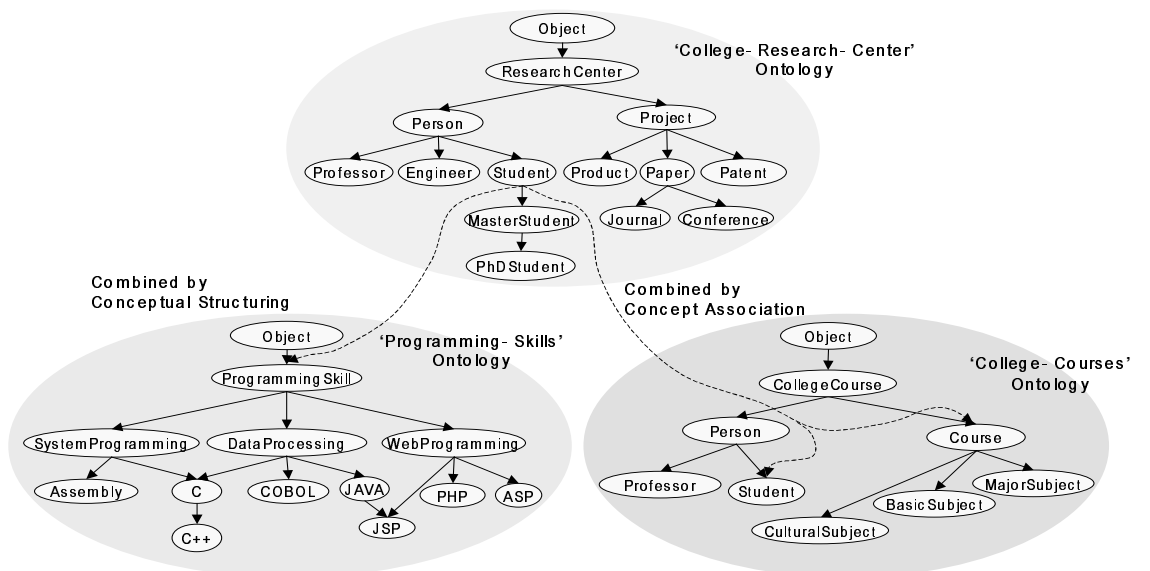

(a) Concept Hierarchies

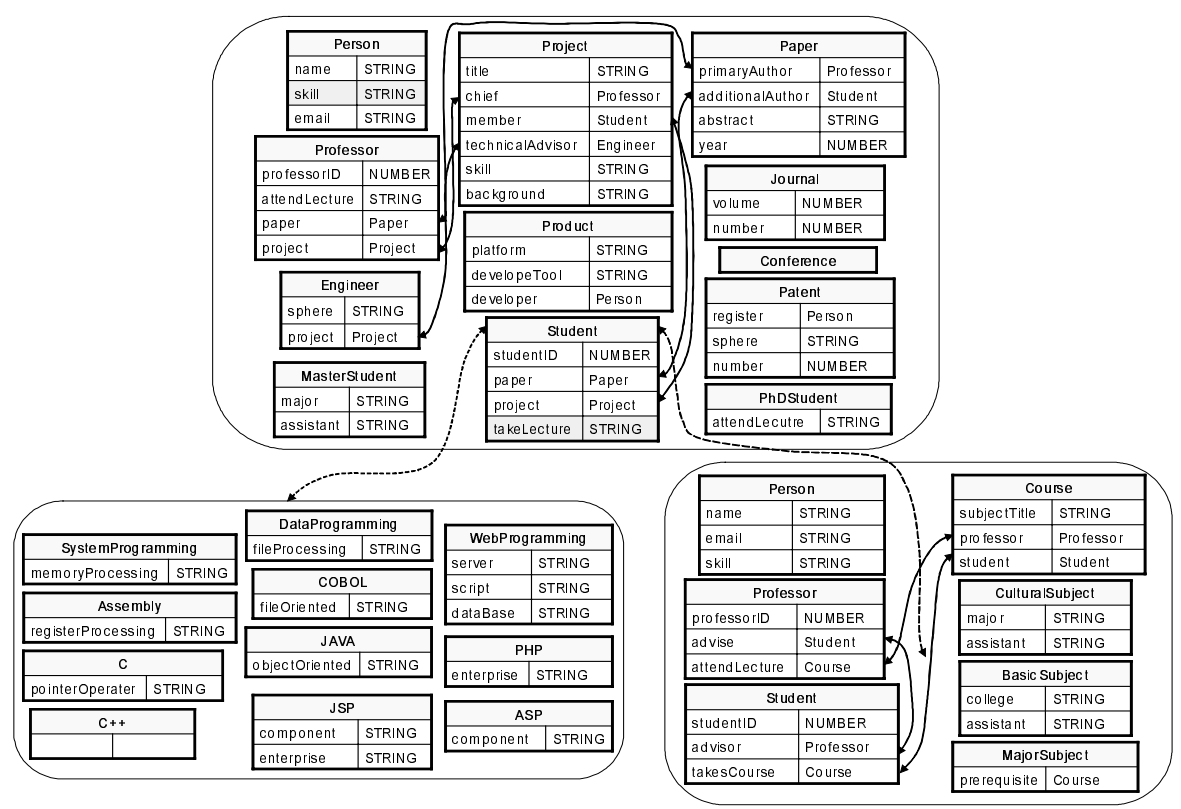

(b) Attributes of Concepts

Fig. 1. Ontology Combination

Combined expansion by conceptual structuring: Conceptual structuring is generalized to include other ontology. By combining 'College-Research-Center' and 'Programming-Skills' by the following rules, query on attribute 'skill' of concept 'Person' can include skill ' $\mathrm{C}++$ '.

FORALL Obj1, Obj2

Obj1 : C -> Obj2 : Student[skill ->> Obj1]

FORALL Obj1, Obj2

Obj1 : ProgrammingSkill -> Obj2 : Student[skill ->> Obj1] 
Combined expansion by concept association: Concept association is also generalized to include other ontology. By combining 'College-Research-Center' and 'College-Courses' by the rule given below, concepts 'Student' and 'Course' can be associated for inter-ontology query expansion.

FORALL Pers1, Cour1

Cour1 : Course[student ->> Pers1] <-> Pers1 : Student[takesCourse ->> Cour1]

\section{Semantic XML Filtering Application}

Semantic XML filtering effect is tested by the XML document in Table 1. By conceptual structuring and concept association, queries '//Student[name="Kim"]' and '//Student[project]' are expanded to '//(Student I MasterStudent I PhDStudent)[name="Kim"]' and '//Project[member]' respectively.

Table 1. XML Document Example

\begin{tabular}{|c|c|}
\hline$<$ ResearchCenter $>$ & $<$ takesLecture $>\mathrm{C}</$ takeLecture $>$ \\
\hline$<$ Person> & $<$ project $>$ Home Page Production \\
\hline$<$ Professor $>$ & $</$ project $>$ \\
\hline$<$ name $>$ Kong $<$ /name $>$ & $</$ Student $>$ \\
\hline <email>yhkong@sch.ac.kr</email> & $</$ Person $>$ \\
\hline$<$ attendLecture $>$ Artificial Intelligence & $<$ Project $>$ \\
\hline$</$ attendLecture $>$ & $<$ title $>$ Semantic XML Query $<$ title $>$ \\
\hline$<$ project $>$ Laser Slice $<$ /project $>$ & $<$ chief $>$ Kong $</$ chief $>$ \\
\hline$<$ project $>$ Semantic XML Query & $<$ member $>$ Lee $<$ /member $>$ \\
\hline$</$ project $>$ & $<$ skill $>$ Java $<$ /skill $>$ \\
\hline$</$ Professor $>$ & $<$ background $>$ XML $<$ /background $>$ \\
\hline$<$ PhDStudent $>$ & $<$ /Project $>$ \\
\hline$<$ name $>$ Kim $<$ /name $>$ & $<$ Project $>$ \\
\hline <email>mskim@sch.ac.kr</email> & $<$ title $>$ Home Page Production</title $>$ \\
\hline$<$ skill $>$ C $++</$ skill $>$ & $<$ chief $>$ Kong $</$ chief $>$ \\
\hline$<$ skill $>$ Open GL</skill $>$ & $<$ member $>$ Kim $<$ /member $>$ \\
\hline$<$ takeLecture $>$ Artificial Intelligence & $<$ member $>$ Shin $<$ /member $>$ \\
\hline$</$ takeLecture $>$ & $<$ Project $>$ \\
\hline $\begin{array}{l}<\text { attendLecture }>\text { Assembly } \\
</ \text { attendLecture }>\end{array}$ & $</$ ResearchCenter $>$ \\
\hline$<$ project $>$ Laser Slice</project $>$ & $<$ Course $>$ \\
\hline$</$ PhDStudent $>$ & $<$ BasicSubject $>$ \\
\hline$<$ MasterStudent $>$ & $<$ subjecTitle $>$ C $<$ /subjectTitle $>$ \\
\hline$<$ name $>$ Lee $<$ /name $>$ & $<$ professor $>$ Kong $<$ /professor $>$ \\
\hline <email>kslee@sch.ac.kr</email> & $<$ student $>$ Shin $<$ /student $>$ \\
\hline$<$ skill $>$ C $</$ skill $>$ & $</$ BasicSubject $>$ \\
\hline$<$ skill $>$ Java $</$ skill $>$ & $<$ MajorSubject $>$ \\
\hline$<$ takeLecture $>$ Artificial Intelligence & $<$ subjectTitle $>$ Home Page Production \\
\hline$</$ takeLecture $>$ & $</$ subjecTitle $>$ \\
\hline$<$ project $>$ Semantic XML Query & $<$ professor $>$ Chun $<$ /professor $>$ \\
\hline$</$ project $>$ & $<$ student $>$ Kim $</$ student $>$ \\
\hline$</$ MasterStudent $>$ & $<$ student $>$ Lee $<$ /student $>$ \\
\hline$<$ Student $>$ & $</$ MajorSubject $>$ \\
\hline$<$ name $>\operatorname{Kim}<$ /name $>$ & $<$ Course $>$ \\
\hline$<$ skill $>$ ASP $<$ /skill $>$ & \\
\hline
\end{tabular}


Query '//Student[skill="C']' is expanded to '//(Student I MasterStudent I PhDStudent)[skill="C”, “C++"]' by combining 'Programming-Skills'. And query //Student[takeLecture] is expanded to //(Course | BasicSubject | MajorSubject)[student] by combining 'College-Courses'. As seen in Fig. 2, the expanded queries by ontology combination successfully filter out the necessary XML documents which were not possible to search before the expansion.

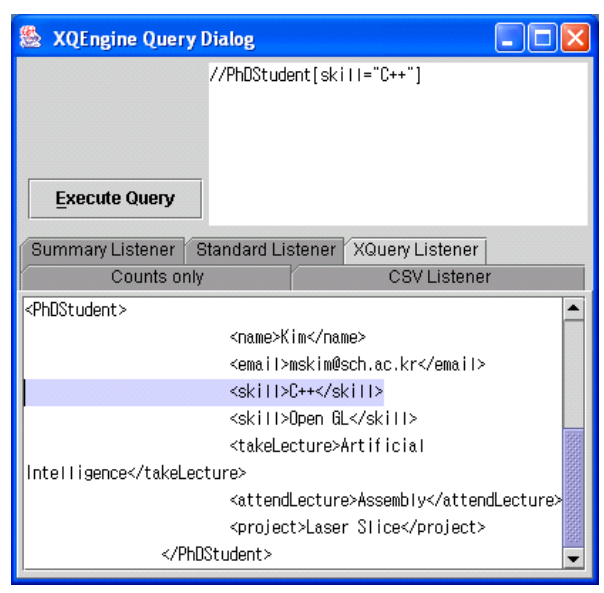

(a) Result of Query //Student[skill="C']

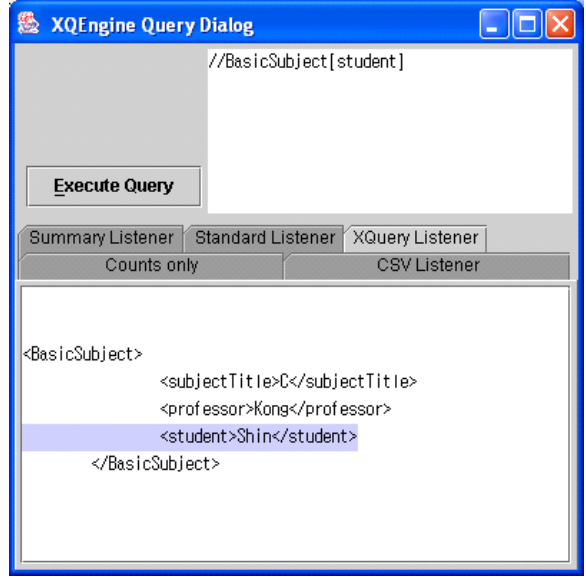

(b) Result of Query //Student[takeLecutre]

Fig. 2. XML Filtering by Ontology Combination

\section{Conclusions}

To filter out semantic information from various forms of XML documents and to be effective to a large domain application, a semantic XML filtering method expands XML queries by conceptual structuring and association in inter-ontology as well as inter-ontology. Experimental result showed that the XML filtering method effectively extracts important information which was undetected before.

\section{References}

1. Fensel, D., Angele, J., Decker, S., Erdmann, M., Schnurr, H.P., Studer, R., Witt, A.: On2broker: Lessons Learned from Applying AI to the Web. Institute AIFB. International Journal of Cooperative Information Systems, Vol. 9. No. 4 (2000) 361-382

2. Erdmann, M., Studer, R.: Ontologies as Conceptual Models for XML Documents. In: Proceedings of the 12th Workshop for Knowledge Acquisition, Modeling and Management (KAW'99), Banff, Canada (1999)

3. Heflin, J., Hendler, J.: Semantic Interoperability on the Web. In Proceedings of Extreme Markup Languages 2000. Graphic Communications Association (2000) 111-120 